\title{
Penser un langage du vivant pour penser les possibilités d'un langage extraterrestre
}

\section{To think a common language of living beings in order to think about the possibilities of an extraterrestrial language}

\author{
Béatrice Fracchiolla ${ }^{1}$ \\ ${ }^{1}$ Professeure en sciences du langage, UMR 8238 LEGS et CREM EA3476
}

\begin{abstract}
RÉSUMÉ. La possibilité d'un langage partagé par des êtres vivants sur des exoplanètes pose la question philosophique et fondamentale de ce qui constitue la communication, un langage, voire une langue. Elle pose la question du sens partagé en même temps que celle d'une intention de communiquer. Nous postulons ici, avant toute chose, la sensorialité du langage, lié à sa perceptibilité, quelle qu'elle soit, et une approche ondulatoire, voire vibratoire, qui semble universelle.

ABSTRACT. The possibility of a language shared by living beings on exoplanets raises the philosophical and fundamental question of what constitutes communication, or even a language. It raises the question of shared meaning at the same time as that of an intention to communicate. We postulate here, above all, the sensoriality of language, linked to its perceptibility, whatever it may be, and an undulatory, even vibratory approach, which seems universal.

MOTS-CLÉS. langage, sensorialité, interactions, communications interespèces, systèmes de signes.

KEYWORDS. language, sensoriality, interactions, interspecies communications, sign systems.
\end{abstract}

\section{Le langage comme sens partagé(s)}

La question posée par l'existence d'un langage ${ }^{1}$ est en réalité celle d'un sens partagé2 ${ }^{2}$ Comment le sens se construit-il entre des êtres vivants qui communiquent ? comment un sens se manifeste-t-il auprès de ses destinataires ou, juste, récepteurs ? Comment est-il compris ? de manière univoque ou équivoque ? Par quel canaux, biais, médiums, etc. arrive-t-il à destination? Comment, sur notre planète Terre, le sens existe-t-il anthropologiquement, dans les échanges interinvididuels, en dehors d'un système strictement linguistique, grammaticalement décrit, déjà, chez nous, humains terrestres? Et qu'apporte de spécifique une mise en langue par un système linguistique ? Les interactions humaines d'avant la parole articulée ${ }^{3}$ (selon un système codifié répertorié) que ce soit chez les petits enfants ou dans l'histoire de l'humanité posent la question des protolangages, qui préexistent toujours aux langues ${ }^{4}$. La notion de protolangage permet de questionner l'apparition même du sens, toujours d'abord interactionnel - le sens n'a de sens, d'une certaine manière, qu'en transmission pour signifier à un autre humain, vivant, auquel on s'adresse ${ }^{5}$. Les signes et canaux paraverbaux nous nourrissent également de signifiés. Le signifié nait et se communique d'abord de manière sensorielle, par l'olfaction, la vue, le toucher, l'ouïe, le goût. Le sens advient de l'interprétation qui est faite par le cerveau, dans la réception, de ces nombreux signaux que la langue

1 Dessalles Jean-Louis, Pascal Picq \& Bernard Victorri, 2010, Les Origines du langage, Paris, Éditions du Pommier.

2 Cislaru, Georgeta, et Vincent Nyckees, 2019, Le partage du sens. Approches linguistiques du sens commun, Londres, ISTE éditions.

3 Oudeyer Pierre-Yves, 2013, Aux sources de la parole : Auto-organisation et évolution, Paris, Odile Jacob.

4 Bickerton D., 1990, Language and Species, University of Chicago Press.

5 Hombert Jean-Marie \& Lenclud Gérard, 2013, Comment le langage est venu à l’homme, Paris, Fayard. 
ne fait finalement que tenter de traduire en mots - et auxquels la linguistique ne s'intéresse historiquement pas, mais les sciences du langage, oui. Or, pragmatiquement, toute traduction constitue une certaine forme de perte du sens initial. Nous passons d'un système d'interprétation multimodal, multisensoriel, «augmenté » dirions-nous aujourd'hui, à une projection représentation « à plat » linéaire, à travers des mots, de ce ressenti, en particulier à l'écrit - l'oral conservant une partie de cette multi modalité/sensorialité. Dans ces conditions, à quoi ressemblerait un langage extraterrestre?

\section{A la base de tout langage il y a un signal}

Extraterrestre ou pas, végétal, animal ou humain, la question du langage pose d'abord celle de la communication et d'une possible intention (comprise ou non, connue ou non) d'une entité lambda à produire un signal $^{6}$. Sur Terre, les recherches les plus récentes vont dans le sens d'une meilleure compréhension des communications interespèces ${ }^{7,8}$. Dans l'espace, les humains envoient des signaux radio à l'aide de leurs sondes à destination de potentielles vies extraterrestres susceptibles de les recevoir. Dans les deux cas, la base du signal est vibratoire : un signal est provoqué par un mouvement volontaire ou involontaire. Sur Terre, nous appelons cela des ondes, des bruits, des cris, de la musique, des langues... si un être vivant est là pour les recevoir, on pourra parler de communication. Et, certainement, dans l'espace aussi. La différence est certainement question de distance, ou d'échelle. Sur notre planète, en effet, les êtres vivants cohabitent dans un tel niveau de densité que la réception est effective à tous les coups, par une autre entité vivante, et commandée en quelque sorte, par la gravité. Sur Terre, nos capacités à recevoir des signaux sont en effet essentiellement sensorielles. Cela est vrai aussi pour les animaux. Les abeilles produisent ainsi une danse, visible par leurs congénères, pour signaler les meilleurs endroits où butiner. La production et la réception de signaux se fait donc sur une base d'abord sensorielle, perceptible. Or, imaginer une vie extraterrestre signifie l'imaginer également sur une base sensorielle, au moins a minima. La vibration semble être le type de signal le plus universellement perceptible, y compris à l'échelle de l'univers. Les sons existent en effet dans l'univers sous formes de vibrations électromagnétiques, enregistrés et traduites par la NASA en véritables sons. Mais nous ne pouvons dire ni savoir si la question de l'intentionnalité, telle que nous la comprenons à notre échelle, est en jeu ni même pertinent dans l'organisation ou pas de ces sons/vibrations électromagnétiques ${ }^{9}$.

\section{La base d'un langage interespèce comme base d'un langage extraterrestre}

La question du décryptage et de la compréhension des messages envoyés sous d'autres formes que les langues humaines a longtemps été ignorée, voire niée, y compris sur Terre. On a ainsi jusqu'à très récemment nié les facultés d'un langage structuré aux animaux (équivalent à ce que les langues sont aux humains) pour ne leur accorder que celle de «communication ». De nombreuses expériences et publications en faisant état démontrent chaque jour un peu plus des capacités cognitives beaucoup plus développées que ce que les humains imaginaient, chez nombre d'animaux, $\mathrm{y}$ compris les poissons, les oiseaux, les poulpes ${ }^{10},{ }^{11}$. Ces expériences montrent qu'une

6 Jakobson, Roman, 1963, Essais de linguistique générale, Paris, éditions de minuit.

7 Haraway, Donna, 2007, When Species Meet, Minneapolis, University of Minnesota Press.

8 Hauser, Marc D., 2002, A quoi pensent les animaux ?, Paris, Odile Jacob.

9 Alex Young, physicien du soleil à la Nasa explique comment le son nous relie au Soleil et à d'autres étoiles. https://www.youtube.com/watch?v=CRu_hG3X3bl ; autres sons de l'univers enregistrés par la NASA :

https://www.nasa.gov/connect/sounds/index.html

10 Strum, Shirley, 1990, Voyage chez les babouins, Paris, Seuil Points Sciences. 
communication interespèce existe et est possible, puisque les animaux réussissent dans les tâches que les humains imaginent pour eux, afin de comprendre s'ils ont ces capacités. Nous savons aussi que des langages animaux existent, chez les grands singes (Strum, 1990), mais aussi chez d'autres animaux ${ }^{12},{ }^{3}$. Il n'y a aucune raison de penser que ces langages équivalant, au niveau des animaux eux-mêmes, à ce que nous appelons une «langue », ne soient pas tout autant organisés en systèmes de signes que nos langues ne le sont. Or, une organisation en système de signes est ce qui fait une langue chez les humains. La langue des signes est l'exemple humain et le résultat de l'adaptation sensorielle et perceptive de ce qu'est une langue vocalisée, moins le sens de l'ouïe. En ce sens, un langage extraterrestre serait sans doute lui aussi sensoriel, mais son type dépendrait du degré de développement sensoriel de ces êtres extraterrestres, comme de leur degré d'évolution cognitif. A quoi ressemblerait un langage fondé uniquement sur la vue, ou le toucher, ou l'odorat ? ${ }^{14}$ Différents humains ayant expérimentés diverses déficiences de naissance ou acquises ont mis en pratique et développé de nouvelles stratégies pour communiquer, comme l'a montré par exemple Dominique Bauby $^{15}$ atteint du locked-in-syndrom ${ }^{16}$. Il est tout à fait envisageable que nous, humains, n'ayons en réalité développé que très peu de sensorialité et qu'en réalité un langage extraterrestre pourrait être beaucoup plus développé que ceux que nous connaissons et imaginons, et que des perceptions d'un autre type, qui nous est inconnu, existe. Des études récentes montrent en effet que les humains possèdent également quatre autres sens supplémentaires : la magnétoréception ${ }^{17}$, l'intéroception ${ }^{18}{ }^{19}$, extéroception et l'écholocalisation ${ }^{20}, 21$. Quatre sens qui sont vraisemblablement partagés par la

11 Morizot, Baptiste, 2020, Manières d'être vivant : Enquêtes sur la vie à travers nous, Paris, Actes sud.

12 Fracchiolla, Béatrice (ed.), 2006, « Les origines du langage et des langues », Marges Linguistiques, $n^{\circ} 11$.

13 Saltzen, Eric A., 2006, « From Calls to Words : How ethology can bridge the divide » in Béatrice Fracchiolla (éd.) L'origine du langage et des langues, Marges Linguistiques, $n^{\circ} 11$, mai 2006, p.200-217.

14 Zemp, Hugo \& Christian Kaufmann, 1969, "Pour une transcription automatique des « langages tambourinés » mélanésiens (Un exemple kwoma, Nouvelle Guinée) ", L’Homme, 9/2, p.38-88.

15 Le scaphandre et le papillon, 1997.

16 II s'agit d'un trouble neurologique rare caractérisé par une paralysie complète des muscles volontaires dans toutes les parties du corps, à l'exception de ceux qui contrôlent les mouvements oculaires. Elle peut résulter d'une lésion cérébrale traumatique, de maladies du système circulatoire, de maladies qui détruisent la gaine de myéline entourant les cellules nerveuses ou d'une surdose de médicaments. Les personnes atteintes du syndrome d'enfermement sont conscientes et peuvent penser et raisonner, mais sont incapables de parler ou de bouger. Le trouble laisse les individus complètement muets et paralysés. La communication peut être possible avec des mouvements oculaires clignotants.

17 Wang, Connie X. , Isaac A. Hilburn, Daw-An Wu, et ali., 2019, « Transduction of the Geomagnetic Field as Evidenced from alpha-Band Activity in the Human Brain », eNeuro 18 March 2019, 6 (2)

18 Chen, Wen G. Dana Schloesser, Angela M. Arensdorf,1 et ali., 2021, « The Emerging Science of Interoception: Sensing, Integrating, Interpreting, and Regulating Signals within the Self », trends in Neurosciences - special issue : The Neuroscience of Interoception, Cell Press, January, Vol.44, Nº1.

19 Petzschner, Frederike H. , Sarah N. Garfinkel, Martin P. Paulus, Christof Koch and Sahib S. Khalsa, 2021, « Computational Models of Interoception and Body Regulation ", Trends in Neurosciences, Special Issue: The Neuroscience of Interoception, CE Press, january, vol.44, $\mathrm{N}^{\circ} 1$

20 Norman L, Dodsworth C, Foresteire D, Thaler L, 2021, « Human click-based echolocation: Effects of blindness and age, and real-life implications in a 10-week training program », PLoS ONE 16(6): e0252330. https://doi.org/10.1371/

journal.pone.0252330

21 Tonelli Alessia, Claudio Campus, Monica Gori, 2020, «Early visual cortex response for sound in expert blind echolocators, but not in early blind non-echolocators », Neuropsychologia, 147 (2020) 107617 
plupart sinon toutes les autres entités terrestres vivantes, mais avec des compétences ou des potentialités d'utilisation variées en fonction des individus. Aujourd'hui, les travaux récents et en cours sur la transe cognitive auto-induite ${ }^{22}$ et le langage, nommé «protolangage », pratiqué par les transeur-euses et sur lequel j'ai initié moi-même une recherche, renforce cette hypothèse de la question vibratoire et de son rôle fondamental dans la production et la sensorialité perceptive, en particulier, chez les êtres humains, par le medium de la voix ${ }^{23}$.

\section{La vibration comme langage universel ?}

Mises bout à bout, ces différentes informations conduisent à faire l'hypothèse que si des vies extraterrestres existent, dans l'univers, d'une part elles auraient nécessairement développé une capacité de langage quelconque, et d'autre part, celle-ci serait vraisemblablement liée à l'émission d'un signal ondulatoire, voire vibratoire. Après, le niveau de structuration des messages émis serait nécessairement codépendant du niveau d'évolution de cette vie, car il est certain que le langage des bactéries n'a rien à voir avec une langue articulée quelconque. On pourrait même se prendre à imaginer (et pourquoi pas ?) que le coronavirus soit un type de vie extraterrestre en train de coloniser l'ensemble des organismes humains de notre planète. Ou alors, qu'il soit lui-même, comme virus, un médium de communication développé par des vies extraterrestre pour tenter de communiquer avec, ou comprendre les humains. Car, fondamentalement, notre propre perception du monde, toujours anthropocentrée, manque souvent d'imagination.

22 La transe auto-induite a été initiée par Corine Sombrun, formée à la transe chamanique, musicologue, musicienne, compositrice et autrice, s'est à la fois investie auprès des chercheurs et prêtée comme sujet volontaire à la recherche scientifique pour isoler certains sons qui permettent à la personne d'entrer en transe, et être en mesure de l'auto-induire (sur demande et sans mouvements parasites) afin de pouvoir en étudier le phénomène au niveau neurophysiologique. 